\title{
The Optimistic Adaptive Modulation Technique for Future Wireless Communication
}

\author{
${ }^{1}$ Kugalur Muthusamy Palaniswamy, \\ ${ }^{2}$ Veerappa Gounder Palanisamy and ${ }^{1}$ Kugalur Muthusamy Palaniswamy \\ ${ }^{1}$ Department of Electronics and Communication Engineering, \\ EXCEL Engineering College, Komarapalayam, Tamilnadu, India \\ ${ }^{2}$ Veerappa Gounder, Palanisamy, Principal, Information and Communication Engineering, \\ INFO Institute of Engineering, Coimbatore, Tamilnadu, India
}

Received 2012-07-04, Revised 2013-01-20; Accepted 2013-05-13

\begin{abstract}
The Future Wireless Communication requires $100 \mathrm{Mbits} / \mathrm{s}$ Data rate, streaming audio/video, asymmetric access/multiple access, Adaptive modulation and coding, dynamic packet arrangement, smart antenna adaptation andIPv6 facility to enhance error free communication. Various digital modulation techniques have been taken for simulation and bit error rate is calculated in both MC-CDMA System and OFDMA system. The continuous phase modulation techniques ensures large bandwidth, high data rate and error free data communication. The convolution coder is mainly chosen to facilitate the Wireless communication. The output from serial to parallel converter is inhibited to various Adaptive modulation schemes like M-ary PSK, Mary QAM, M-ary CPM and M-ary MHPM systems. The modulated output is then fed to MC-CDMA System and OFDMA system in fast fading Rayleigh environment. The BER performance and SNR ratio are observed for both the MC-CDMA system and OFDMA System inhibiting various modulation schemes and the corresponding simulation result are plotted using Mat lab and Simulink software. The outputs of various modulation schemes are plotted for both OFDMA and MC-CDMA systems. It is found that MHPM is found to be the optimistic Adaptive modulation technique for future Wireless communication.
\end{abstract}

Keywords: MC-CDMA-Multi Carrier Code Division Multiple Access, OFDMA-Orthogonal Frequency Division Multiple Access, PSK-Phase Shift Keying, QAM-Quadrature Amplitude Modulation, CPM- Continuous Phase Modulation, MHPM-Multi-Hop Phase Coded Modulation

\section{INTRODUCTION}

\subsection{Performance}

The Future mobile (4G) communication feature extremely high quality video with large bandwidth, high data rate and error free data communication. It also enables wireless download with speed of up to $100 \mathrm{mbps}$.

\subsection{Interoperability}

Since there are many standards in $3 \mathrm{G}$ Networks, It is difficult to roam and interoperate across networks. It is overcome by providing a Global standard and provides compatibility between various standards.

\subsection{Networking}

The Future Mobile (4G) Communications Network will be a Hybrid Network which utilize both wireless Local Area Access Network (LAN) concept and Wide Area Access Network (WAN) in design process. The $4 \mathrm{G}$ network enhances Internet Protocol version 6 (IPv6) and intended to succeed IPv4.

\subsection{Data Rate}

4G facilitates transmission speed of more than 100 Mbits/s and offers higher bandwidth within the reach of LAN.

Corresponding Author: Kugalur Muthusamy Palaniswamy, Department of Electronics and Communication Engineering, EXCEL Engineering College, Komarapalayam, Tamilnadu, India 


\subsection{Convergence}

The Future Wireless Communication Utilizes IP in full form with converged voice and data capability.

\subsection{Cost}

It will be cheaper than the existing $3 \mathrm{G}$ System as it utilizes the most advanced technology in the existing Network. It does not require the operators to purchase extra spectrum.

\subsection{Scalability}

Scalability or the ability to handle increased no of users and diversity of services are easily achieved in the future Wireless communication as the IP core is easily scalable.

IPv6 supports globally unique static IP addresses, which can be used to track a single device's Internet activity. Since the devices are used mostly used by a single user, the device activity is often assumed to be equivalent to the user's activity 4g-Encyclobedia, 4g-wikepedia. 2000):

The Composite vision of $4 \mathrm{G}$ includes (Watson,

- $100 \mathrm{Mbits} / \mathrm{s}$ data rate

- Streaming audio/video

- Asymmetric access/multiple access.

- Adaptive modulation/coding

- Dynamic packet arrangement

- Smart antenna adaptation.

- IPv6 facility

\subsection{Section-2}

\subsubsection{Importance of MC-CDMA and OFDMA}

MC-CDMA and OFDMA are considered to be the best suited accessing techniques for future Wireless communication physical layer. It is recognized that future enhancements in Wireless communication includes the Inherent ability to support adhoc based Wireless networks.

Inter Symbol Interference (ISI) which Results due to the delay spread of the signal caused by multipath effects of the received signal is avoided by providing Guard Interval (GI) i.e., Pilot Symbol Insertion either in front end or back end with longer delay than the delay spread.

As the Wireless channel varies rapidly with time to time, the fixed channel modulation system becomes inefficient. Since it uses a higher order modulation format only during good channel condition, Adaptive modulation techniques are best suited to achieve efficient and effective communication over multipath fading transmission channels.

\subsection{A-Subsection}

\subsubsection{Turbo Coder}

The convolution coder i.e., TURBO Coder (High performance forward error correction coder) is selected to meet the dynamic channel capacity for noise free communication. It finds specific use where designers seek to achieve Information transfer over larger bandwidth and enhances latency constrained communication link. The encoder is implemented by sending three sub blocks of bits. The first Sub block is the $\mathrm{m}$ bit block of payload data. The second sub-block is $\mathrm{n} / 2$ parity bits for payload data computed using a Recursive Convolution Code (RSC) and the third sub block is $\mathrm{n} / 2$ parity bits for a known permutation of the payload data is computed using an RSC Convolution Code. The Turbo coder design parameters are given in the Table 1.

\subsection{B-Subsection}

\subsubsection{Modulation Techniques}

The combination of convolution coder, OFDMA system and MC-CDMA system with Adaptive modulation techniques (M-ary QAM, M-ary PSK, Mary CPM and M-ary MHPM) in a free Rayleigh fading channel is taken and Signal to Noise Ratio (SNR) Vs Bit Error Rate (BER) is plotted for up to 32 users using Mat lab and Simulink software.

\subsection{Relative Concept}

If the fixed modulation technique is designed by considering a worst case scenario, the system may be good only for worst case scenario and during other times the system experiences very poor SNR. Hence it is decided to go for Adaptive modulation system with Marray techniques.

Taking advantage of the time varying nature of Wireless channels, the Adaptive modulation scheme varies transmission power, data rate, constellation size, coding and modulation scheme or combination of any of the parameters according to the state of the channel. The Adaptive modulation technique provides a good SNR during normal time and as the system worsens, the modulation scheme change its array accordingly as (Marray-2,4,8,16,32 ....) and tune itself to provide good SNR.

\subsection{C-Subsection}

\subsubsection{OFDMA Vs MC-CDMA Performance}

For fully loaded system the OFDMA surpasses MC-CDMA in the error performance where as for a partially loaded system, MC-CDMA surpasses the OFDMA performance by utilizing its whole diversity of used sub-carriers. 
Table 1. System parameters

\begin{tabular}{ll}
\hline Parameters Specifications & \\
\hline Channel bandwidth $>20 \mathrm{mhz}$ & $5 \mathrm{GHZ}$ \\
Frequency & 1024 \\
No of subcarriers & $25 \mathrm{KHZ}$ \\
Subcarrier spacing & $40 \mu \mathrm{s}$ \\
Portion of symbol & $10 \mu \mathrm{s}$ \\
Cyclic extension duration & $50 \mu \mathrm{s}$ \\
Total symbol duration & \\
Symbol rate $640 \mathrm{ksymbol} / \mathrm{s}$ & \\
Chip rate 20.48 Mchip[s/s & \\
CDMA code Walsh hadamard & \\
Code length 32 chips &
\end{tabular}

Table 2. Channel parameters

\begin{tabular}{ll}
\hline Parameters & Specifications \\
\hline Mobile speed $100 \mathrm{~km}$ per hour & \\
Number of paths Four with Exponential & \\
power distribution & \\
Maximum excess delay $150 \mu \mathrm{s}$ & \\
Decaying factor $10 \%$ of symbol \\
duration
\end{tabular}

Table 3. Turbo coder parameters

\begin{tabular}{ll}
\hline Parameters & Specifications \\
\hline Rate $1 / 3$ & 3 \\
$\begin{array}{l}\text { Constraint length } \\
\text { Interleaving } \\
\text { with block size } 1024 \text { bits } \\
\begin{array}{l}\text { Decoding } \\
\text { hard inputs }\end{array}\end{array}$ & Block interleaving \\
\hline
\end{tabular}

In this case, MC-CDMA can even gain $2 \mathrm{~dB}$ compared to OFDMA and hence it is found that both OFDMA and MC-CDMA are highly suited for multi cellular environment.

\subsection{System Design Concept}

\subsubsection{System Design}

The proposed OFDMA System and MC-CDMA system is designed by using the specified parameters given in the Table 2 below. The assumed frequency is $5 \mathrm{GHz}$ with a channel bandwidth of $20 \mathrm{MHz}$. The no of sub carriers is choosen as 1024 with subcarrier spacing of 25 $\mathrm{kHz}$. The symbol rate is fixed as $640 \mathrm{ksymbols} / \mathrm{s}$. The Walsh hadamard code is taken as PN sequence code containing 32 chips.

\subsection{Chatterjee et al. (2003) Channel Parameters}

The Rayleigh fading channel with a mobile speed of $100 \mathrm{~km} / \mathrm{h}$ (Kilo Meter per hour) is taken and parameters are given in the Table 3 below. Here the signal takes four exponential paths with a decaying factor of $10 \%$ (Steele and Webb, 1991).

\subsection{Coder Design}

The 1/3 Turbo coder with a constraint length of 3 and block interleave with 1024 bits size are taken as design parameters for Coder design and MAP decoding with hard inputs (Chatterjee et al., 2003).

\subsection{D-Subsection}

\subsubsection{Implementation of Adaptive modulation techniques}

Steele and Webb (1991) proposed burst by burst Adaptive Quadrature Amplitude Modulation (QAM) for exploiting the time variant Shannon Channel capacity of narrow band fading channels. The above system sacrificed the bit error rate for getting better fixed data throughput.

Keller and Hanzo (1998) proposed Adaptive modulation techniques with a set of QPSK and QAM modulation Schemes for OFDM duplex transmission where as Wasantha (2000) has proposed an Adaptive COFDMCDMA system with QAM, PSK and MHPM modulation schemes. Wasantha and Fernando (2002), Adaptive modulation improves the system throughput considerably by matching transmitter parameters to timevarying wireless fading channels.

\subsection{Features of Adaptive Modulation}

RF power output has been a major planning aspect for engineers since from the beginning of radio transmission. Undoubtedly important, RF power level is one of the many factors that determine a successful wireless network.

To evaluate and differentiate between various microwave systems and link performance, several key aspects of RF power output. Propagation and antenna parameters such as receiver threshold, modulation type and RF power level are to be considered.

Adaptive modulation schemes and Automatic Transmit Power Control (ATPC) provide point-to-point microwave systems with a high degree of flexibility and ensures better efficiency under changing weather conditions. RF output power can be controlled dynamically so as to ensure the highest power efficiency under changing modulation.

The goal of Adaptive modulation is to improve the operational efficiency of microwave links by increasing network capacity over the existing infrastructure and reduce sensitivity to environmental interference.

Adaptive modulation means dynamically varying the modulation in an errorless manner in order to maximize the throughput under momentary propagation conditions. In other words, a system can operate at its maximum throughput under clear sky conditions and decrease it gradually under rain fade. 


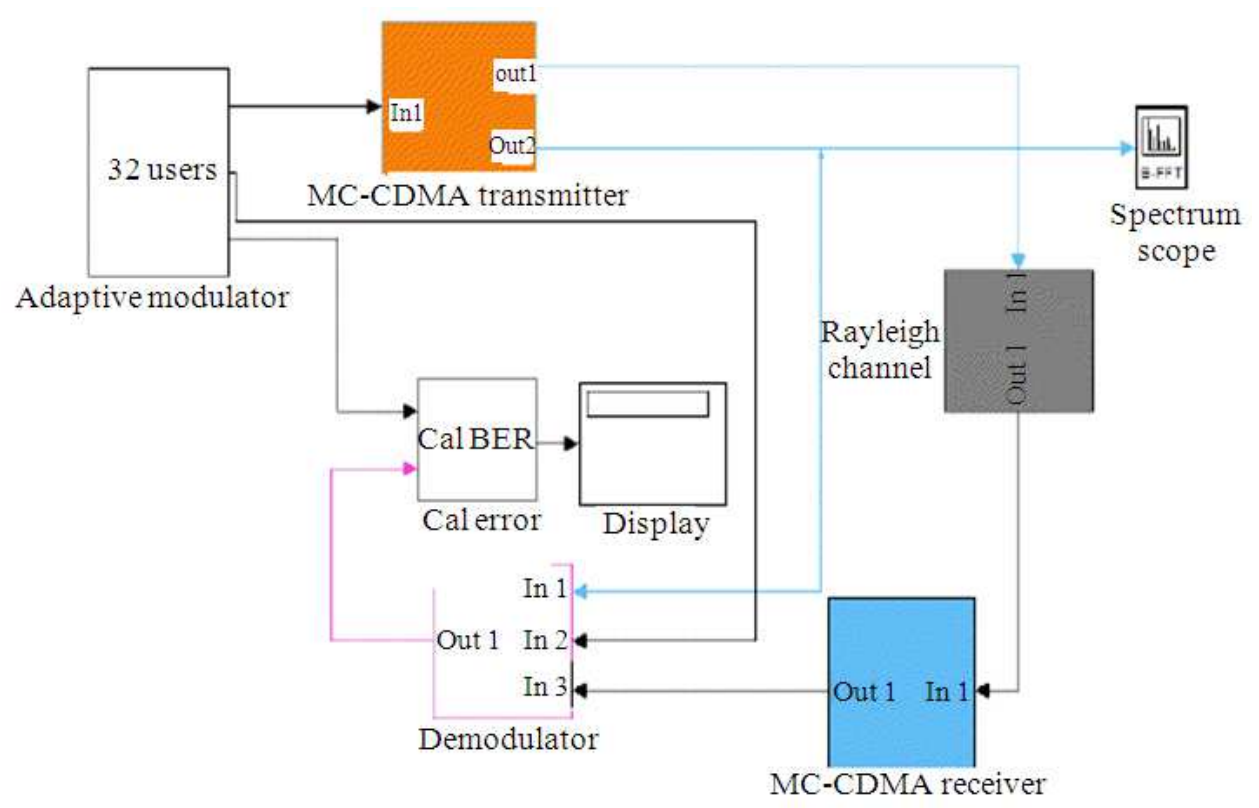

Fig. 1. Proposed system

In the proposed system shown in Fig. 1, data is provided to 32 user channel with TURBO Encoder and Adaptive modulator consisting of M-ary PSK, M-ary QAM, MaryCPM and M-aryMHPM. The modulator output is connected to MC-CDMA transmitter. The spread signal from the MC-CDMA transmitter is transmitted through the Rayleigh fading channel.

In the Receiver, The Received signal is passed through the MC-CDMA Receiver. The output signal from MC-CDMA Receiver is taken to Adaptive demodulator and the demodulator output is taken to the decoder. The decoded data is taken as the output.

\subsection{E-Subsection}

\subsubsection{Channel Quality Estimation}

The impact of channel on the bit error rate performance is analyzed. Channel predictor based on pilot symbol assisted modulation for MIMO Raleigh fading channel is provided. According to the expected channel condition for next time slot, the modulation scheme is selected by the transmitter.

\subsection{Signaling Conditions}

The Receiver has to be intimated properly with the set of demodulator parameters to be decided by the receiver for proper receiving of the signal (Rajwani et al., 2012).

\subsection{F- Developments}

Adaptive modulation improves the system throughput considerably by matching transmitter parameters to timevarying wireless fading channels.

Finally through simulation Result, It is confirmed that MHPM modulation is found to be the best modulation technique for both OFDMA as well as MC-CDMA system.

\subsection{Approach}

- The proposed system is designed for 32 users

- The OFDMA/MC-CDMA Transmission system is opted

- The Adaptive modulation techniques like M-ary PSK, M-ary QAM, M-ary CPM and M-ary MHPM are inhibited

- The Turbo coder is designed for symbol rate of 640 ksymbols/sec

\subsection{Implementation Procedure}

In the present modulation techniques, spread spectrum code division multiple access has reached the level of maturity. The commercial product and operational networks operate with low spectral density and facilitates coexistent with other system. The main drawback of spread spectrum techniques is detection of unauthorized users. 


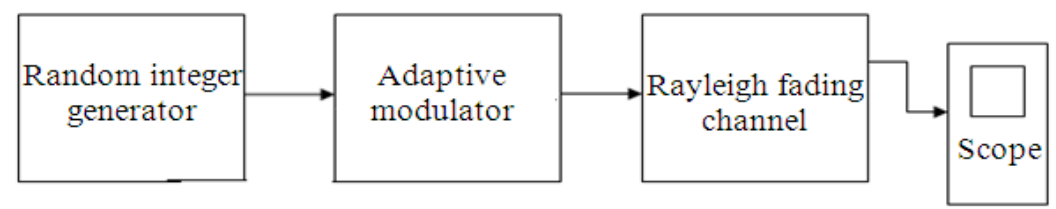

Fig. 2. Simple modulator in Rayleigh fading environment

The drawback can be overcome in OFDM system by implementation of following steps:

- The serial to parallel converted data stream is spread by using the given spreading code and then different subcarriers are modulated with each data stream

- The use of conventional CDMA is not applicable as the data rate goes above $100 \mathrm{Mbits} / \mathrm{s}$ due to severe intercede interference. The solution for the above problem is achieved by finding a new technique called Adaptive modulation technique which reduces both the symbol rate and the chip rate.

- The common point is to change the conventional serial transmission of data/chip stream in to parallel transmission of data/chip symbols over large number of narrowband orthogonal carriers. Hence the chip and bit duration is increased proportionally

The Fig. 2 represents the simple Transmitter with data source, transmitter and channel. Here the Random integer generator is chosen as source to provide necessary data to Adaptive modulator for modulation. The modulated data is spreaded using MC-CDMA transmitter and passed through the Rayleigh fading channel.

Rayleigh fading is used in heavily built-up city centers where there is no line of sight between the transmitter and receiver. The buildings and other objects attenuate, reflect, refract and diffract the signal. In troposphere and ionosphere signal propagation, the particles in the atmospheric layers act as scatterers and this kind of environment may also approximate Rayleigh fading. The Output from Rayleigh fading channel is taken to scope for display. (en.wikipedia.org/wiki/Rayleigh_fading)

The various types of Adaptive modulation schemes selected for transmission system are QPSK QAM,CPM and MHPM system and their descriptions, are listed below (Xiong, 2006).

\subsection{QPSK}

\subsubsection{M-Ary Phase-Shift Keying}

- Phase-Shift Keying (PSK) is a digital modulation scheme that conveys data by changing, or modulating, the phase of a reference signal (the carrier wave)

- Usually, each phase encodes an equal number of bits. Each pattern of bits forms the symbol is represented by the particular phase

- The demodulator, which is designed specifically for the symbol-set used by the modulator, determines the phase of the received signal and maps it back to the symbol it represents and recovers the original data. If the receiver is able to compare the phase of the received signal to a reference signal, such a system is termed coherent PSK (referred as CPSK)

\subsection{QAM}

\subsubsection{QAM}

- It has both an analog and a digital modulation scheme. It conveys two analog message signals, or two digital bit streams by changing (modulating) the amplitudes of two carrier waves, using the Amplitude-Shift Keying (ASK) or amplitude Modulation (AM)

- The two carrier waves, usually sinusoids, are out of phase with each other by 90 degree and are thus called quadrature carriers. The modulated waves are summed and the resulting waveform is the combination of both Phase Shift Keying (PSK) and amplitude-Shift Keying (ASK).

\subsection{Continuous Phase Modulation (CPM)}

- CPM called as constant envelope waveform method is mostly used in wireless modems

- In contrast to other coherent digital phase modulation techniques where the carrier phase abruptly resets to zero at the start of every symbol (example-M-PSK), here in CPM, The carrier phase is modulated in a continuous manner

- As the transmitted carrier power is maintained constant, the phase continuity yields high spectral efficiency and the constant envelope yields high power efficiency 


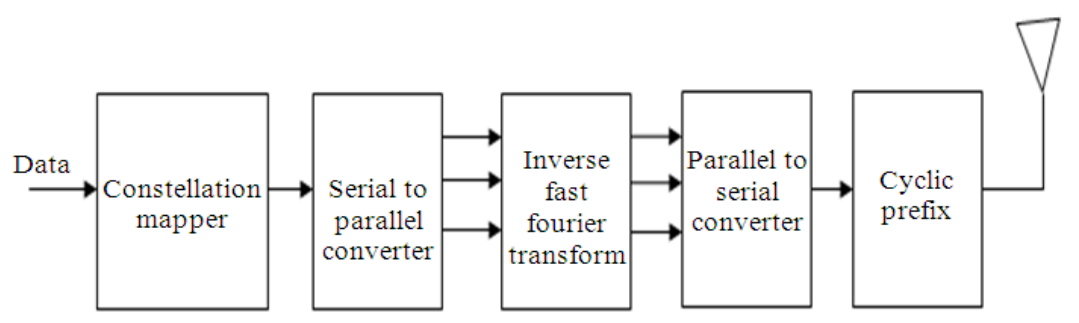

Fig. 3. OFDMA transmitter

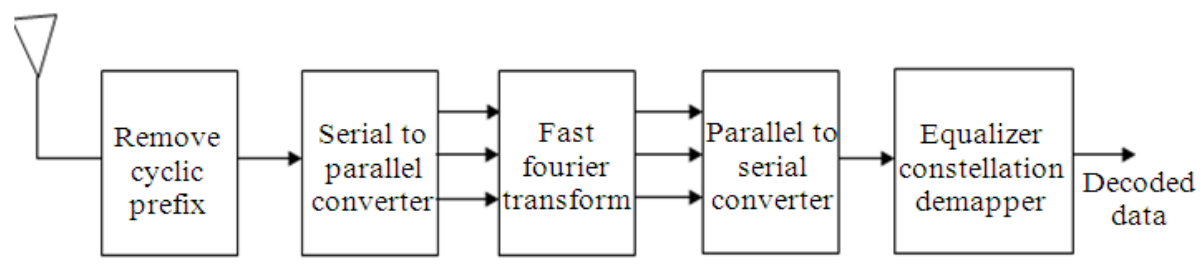

Fig. 4. OFDMA receiver

\subsection{Multi-H Phase Coded Modulation (MHPM)}

- MHPM scheme was first explained in detail by Anderson and Taylor. It is a bandwidth efficient modulation scheme which offers substantial coding gain over conventional digital modulations

- Here modulation indices are used in a prescribed manner such that the transmitted signal has phase slope variation changing from one symbol interval to the next in response to the data symbols being transmitted. The Trellis paths will results in longer minimum Euclidean distances for MHPM schemes provides the coding gain as compared to other conventional Modulation schemes (Watson, 2000)

\subsection{OFDMA and MC-CDMA Implementation}

The serial data is converted to parallel data streams before modulated on to subcarriers. The sub carriers are then sampled at a rate of N/Ts. The samples on each carrier are summed together to form an OFDM sample.

An OFDM sample is generated by an N-Sub carrier OFDM system consists of $\mathrm{N}$ samples and the $\mathrm{m}^{\text {th }}$ sample of an OFDM symbol (Keller and Hanzo, 2000):

$$
\mathrm{X}_{\mathrm{m}}=\sum_{\mathrm{n}-1}^{\mathrm{N}-1} \mathrm{Xne}^{\mathrm{j} 2 \mathrm{xmn} / \mathrm{N}}(0<\mathrm{m}<\mathrm{n}-1)
$$

where, $\mathrm{Xn}=$ Transmitted data symbol on $\mathrm{m}^{\text {th }}$ carrier.

The base band signal thus created is modulated by a carrier to become a band pass signal before transmission:

- The original data is spread using a spreading code and then different sub carriers are modulated with each chip. A fraction of the symbol corresponding to the chip of the spreading code is transmitted through a different sub carrier

- The transmitter and receiver scheme of OFDMA and MC-CDMA differs only in sub carrier allocation, addition and detection of components of $\mathrm{MC}$ CDMA transmission scheme

- The information bit stream of $\mathrm{N}_{U}$ active users are mapped to complex valued data symbols

- In the subcarrier allocation $\mathrm{N}_{\mathrm{d}}$ symbols per user are arranged for each transmission scheme. In case of MCCDMA the $\mathrm{K}^{\text {th }}$ data symbol is multiplied by Walsh Hadamard spreading sequence and provide chips

- The spreading length varies accordingly with maximum no of active user (i.e.) $\mathrm{L}=\mathrm{N}_{\mathrm{U}}$ (max)

- Finally OFDM modulation is performed by using Inverse Fast Fourier Transform (IFFT), which results in addition of Guard Interval (GI) to avoid Intersymbol Interference (ISI) and Interchannel Interference (ICI) in the receiver

- In the receiver side the transmission process is reversed for the detection of original signal

- In MC-CDMA the distortion due to flat fading on each sub channel is compensated by equalization. The coded bits are deinterleaved and finally decoded (Chen and Wang, 2001)

In Fig. 3 and 4, OFDMA, subcarriers are grouped into larger units, referred to as sub channels and these sub channels are further grouped into bursts which can be allocated to wireless users. Each burst allocation can 
be changed from frame to frame as well as within the modulation order. This allows the base station to dynamically adjust the bandwidth usage according to the current system requirements.

In OFDMA transmitter shown in Fig. 3, the incoming data stream is mapped to the corresponding signal constellation according to the modulation scheme. The sample of the transmitted OFDM symbol can be obtained by performing an Inverse Fast Fourier Transform (IFFT) operation on the group of data symbols which are to be sent on orthogonal subcarriers. Similarly, in Fig. 4 the recovery of the data symbols from the orthogonal subcarriers is accomplished by using a Fast Fourier Transform (FFT) operation on the block of received sample.

\subsection{MC-CDMA Transmitter}

In Fig. 5 the incoming data stream is mapped to the corresponding signal constellation according to the modulation scheme. The samples of the OFDM symbol can be obtained by performing an IFFT Operation on the group of data symbols and sent on orthogonal subcarriers (McCormick and Al Susa, 2002). After spreading chips, the user unique PN sequence is added. The result of the summation is a composite sequence. Multi-carrier modulation is achieved by applying IFFT to the summarized signal. Every chip in the composite sequence corresponds to one frequency bin. The circular prefix is attached to every block of $\mathrm{N}$ samples. Finally the signal is filtered in pulse shaping filter.

\subsection{Receiver}

In Fig. 6 the recovery of data symbols from orthogonal subcarriers is achieved by using FFT operation on the block of received symbols.

The receiver consists of many blocks. The output of FFT are fed to $\mathrm{x}$ branches (one branch for each user). An N equalizer and despreading block is applied in every branch to equalize the user's individual channel and multiplication of received signal is achieved by user's unique PN code (Chen and Wang, 2001).

\subsection{OFDMA System: M-ary PSK, M-aryQAM}

M-aryCPM, M-ary MHPM are shown in the following Fig. 7-10.

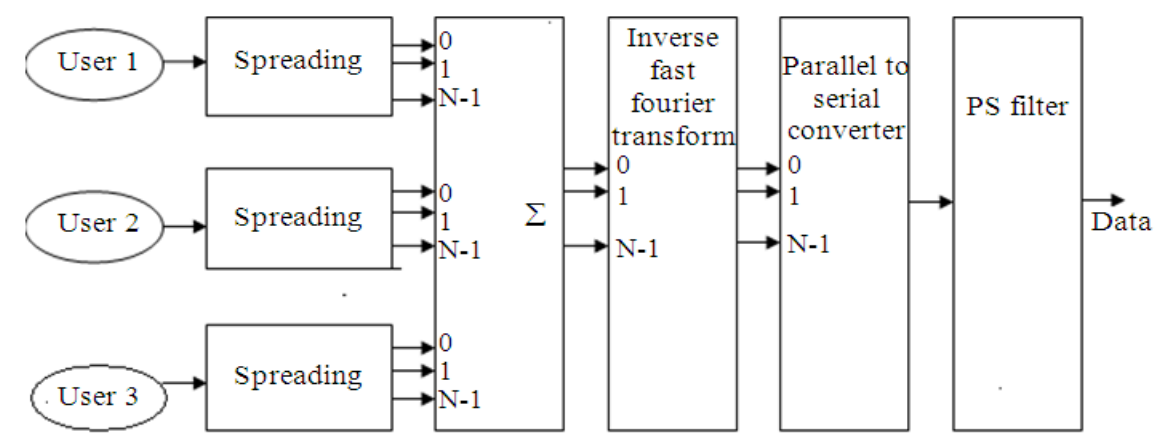

Fig. 5. MC-CDMA transmitter

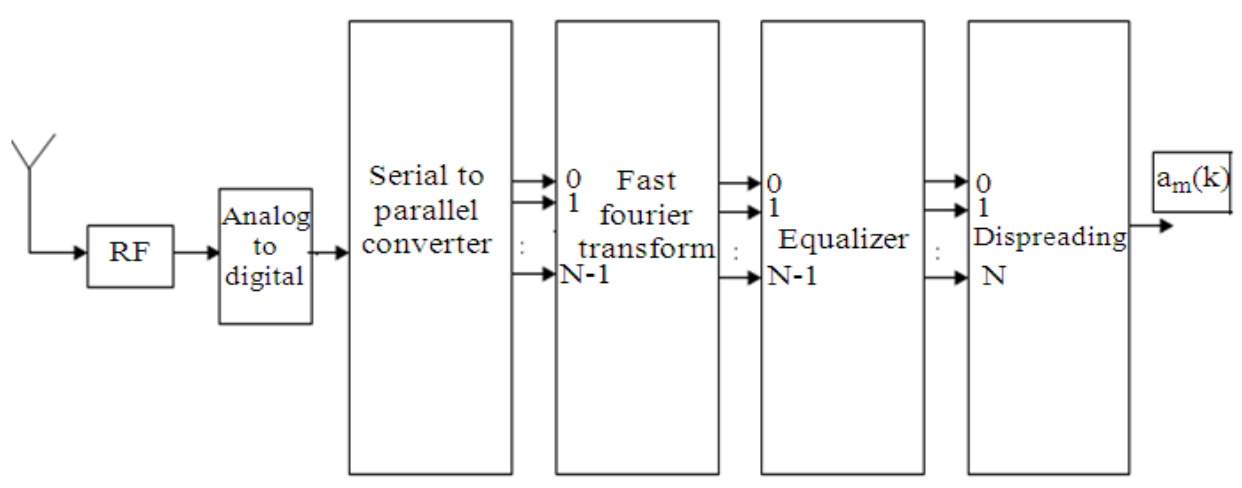

Fig. 6. MC-CDMA receiver 
Kugalur Muthusamy Palaniswamy et al. / Journal of Computer Science 9 (4): 500-513, 2013

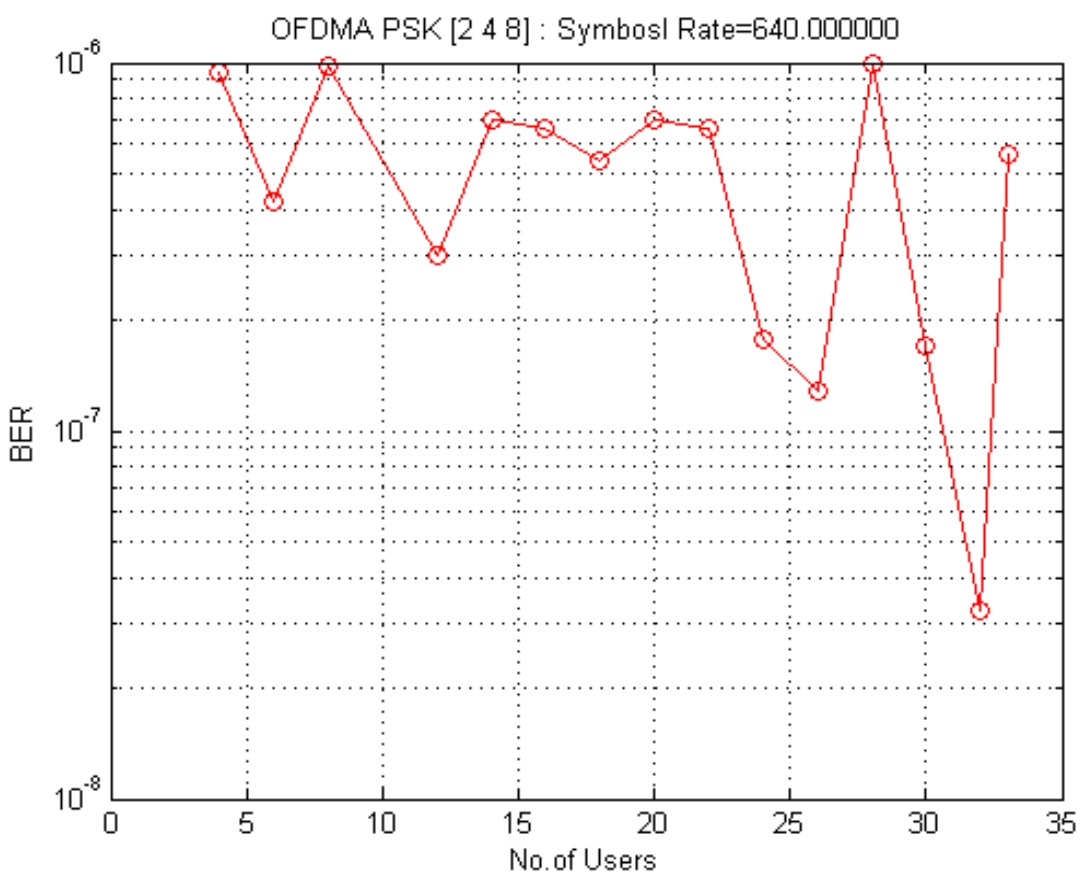

Fig. 7. OFDMA SystemM-ary PSK

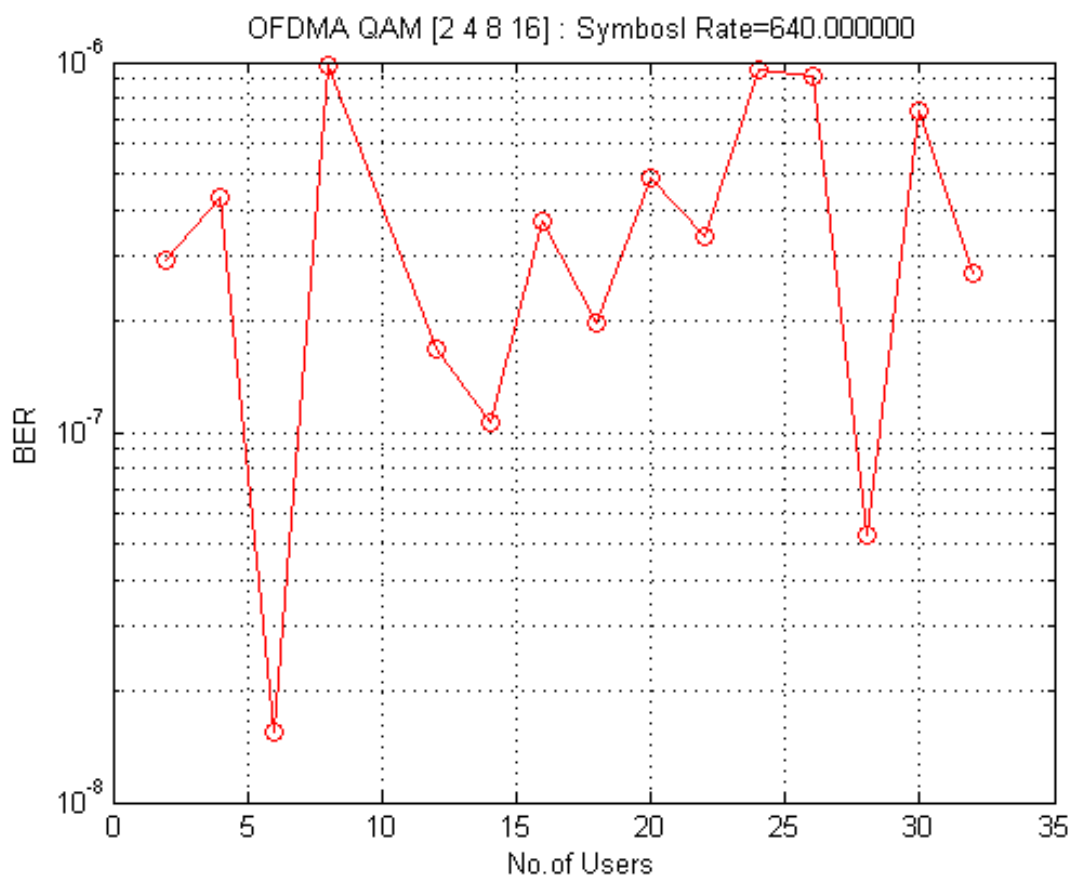

Fig. 8. OFDMA System M-ary QAM 
Kugalur Muthusamy Palaniswamy et al. / Journal of Computer Science 9 (4): 500-513, 2013

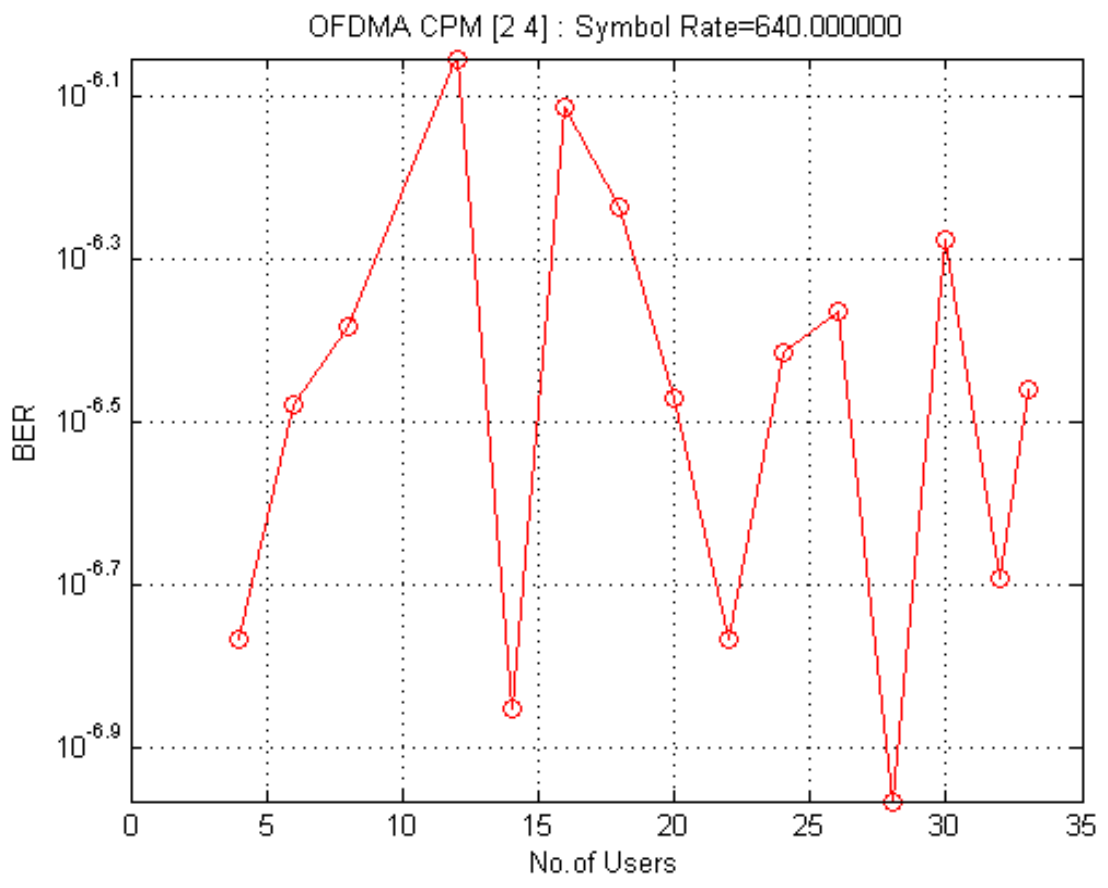

Fig. 9. OFDMA System M-ary CPM

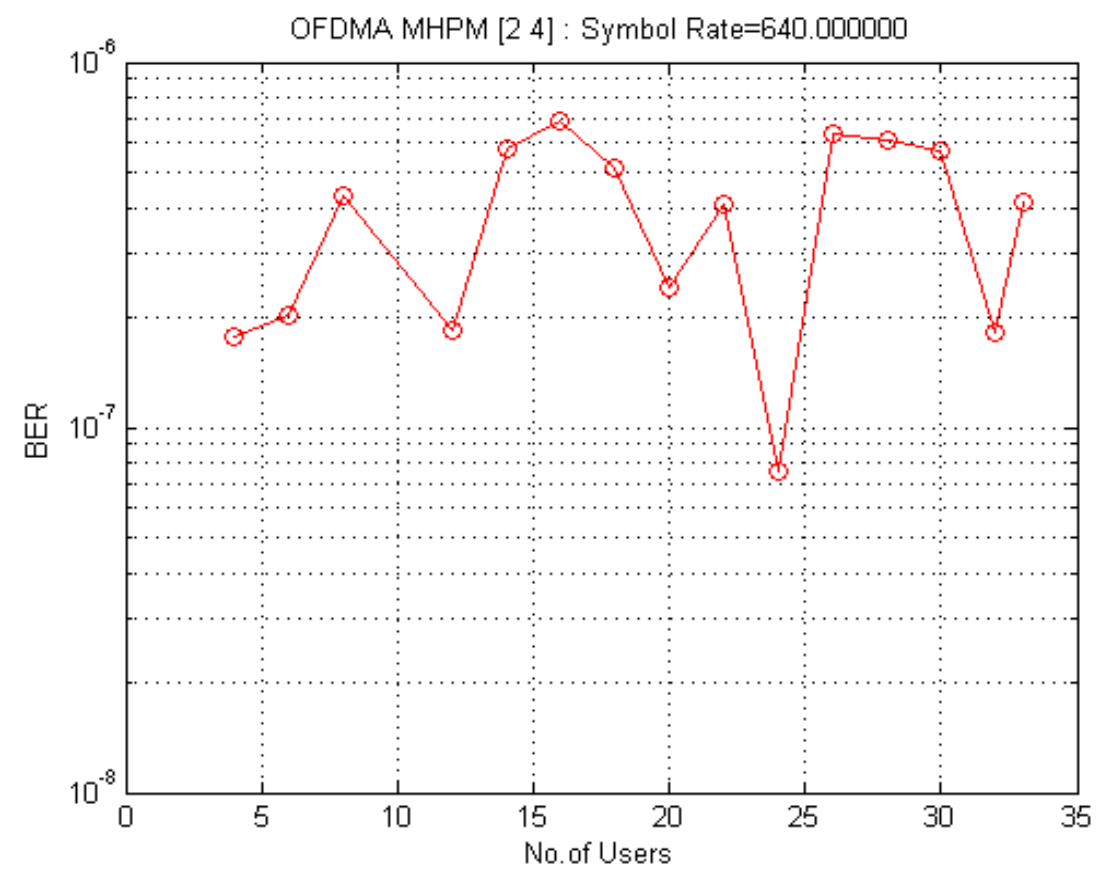

Fig. 10. OFDMA System M-ary MHPM 
Kugalur Muthusamy Palaniswamy et al. / Journal of Computer Science 9 (4): 500-513, 2013

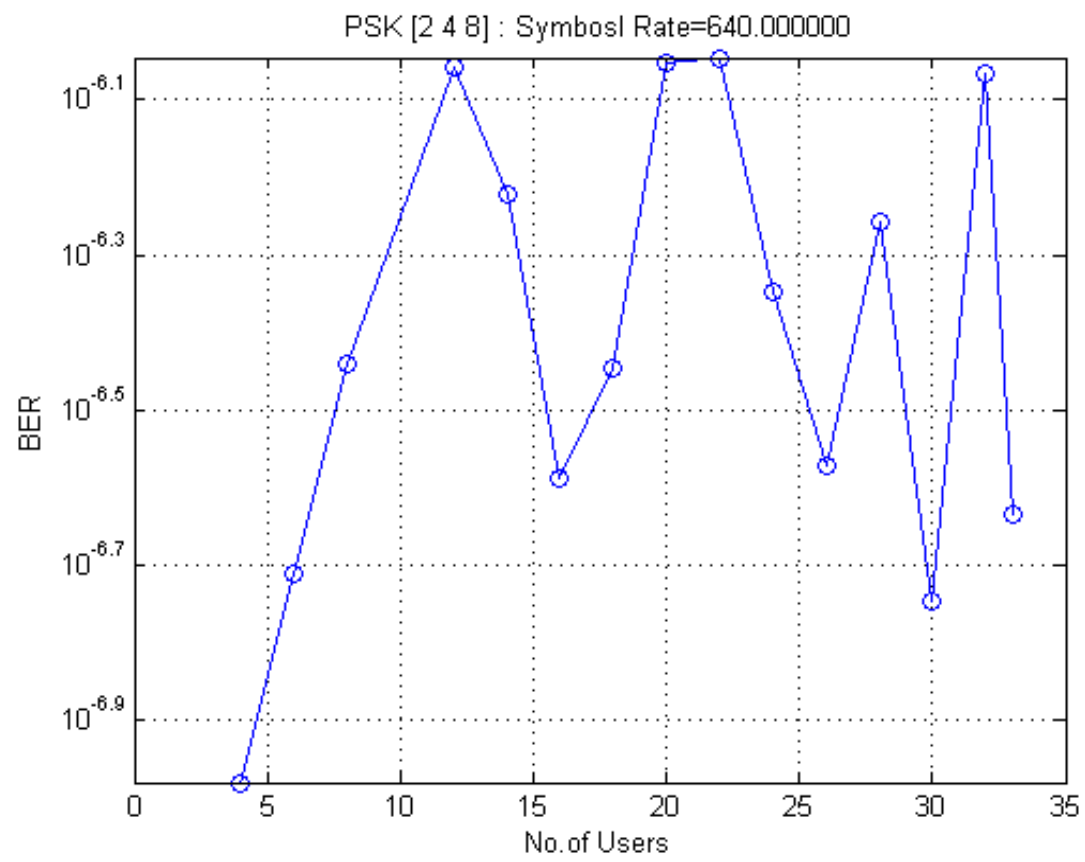

Fig. 11. MC-CDMA System M-ary PSK

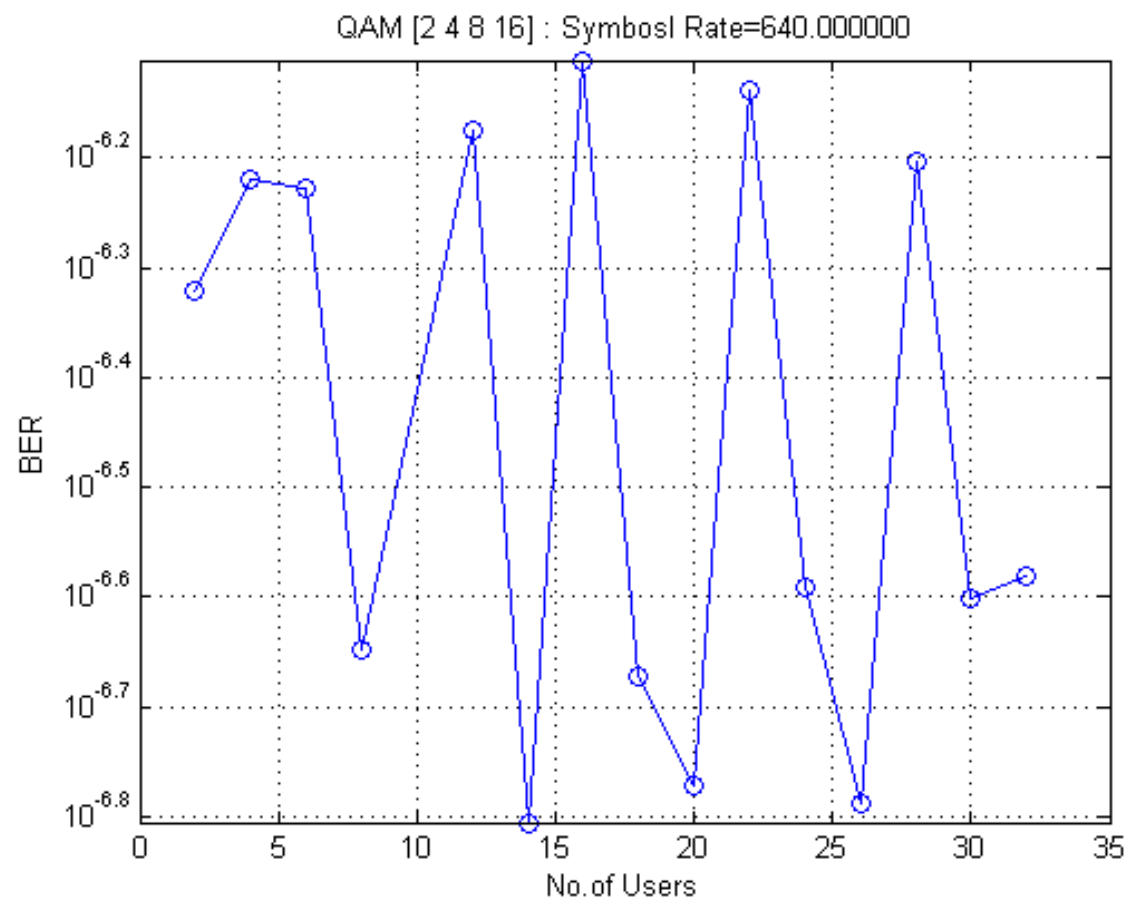

Fig. 12. MC-CDMA System M-ary QAM 
Kugalur Muthusamy Palaniswamy et al. / Journal of Computer Science 9 (4): 500-513, 2013

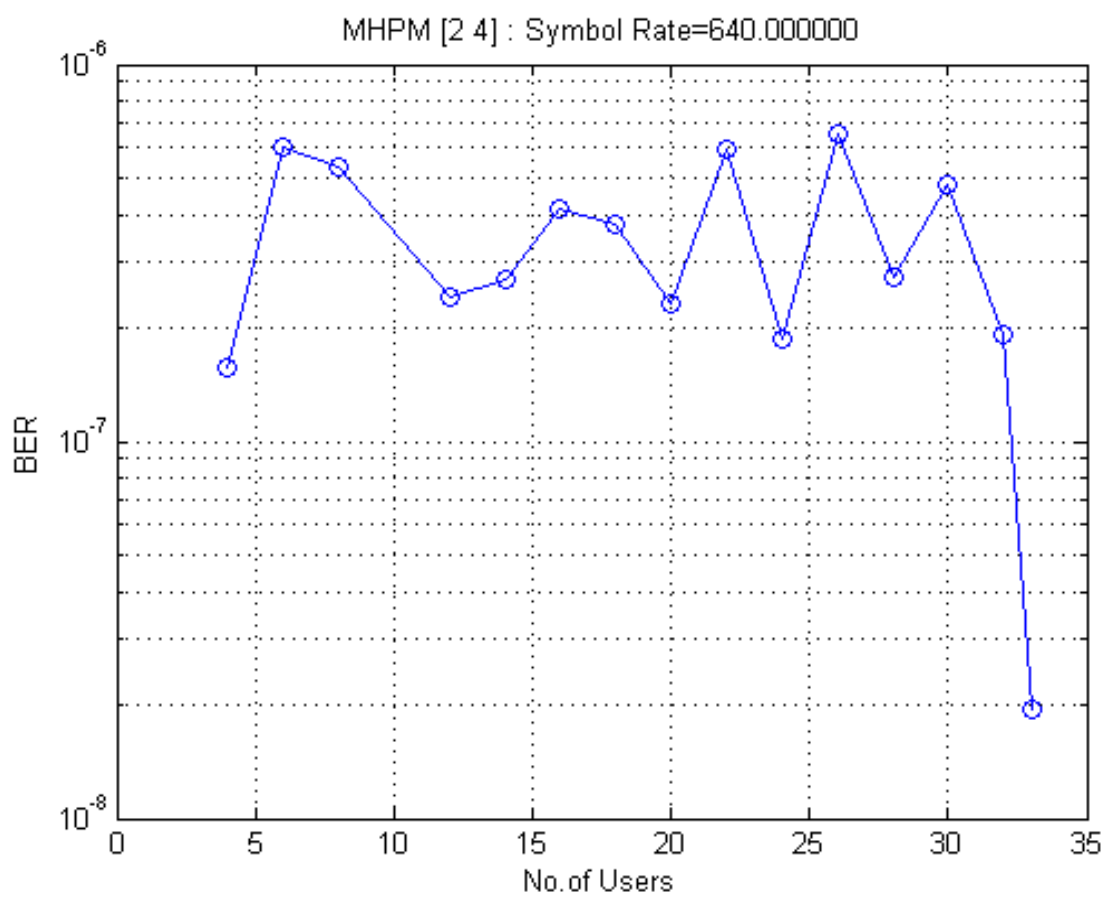

Fig. 13. MC-CDMA system Mary MHPM

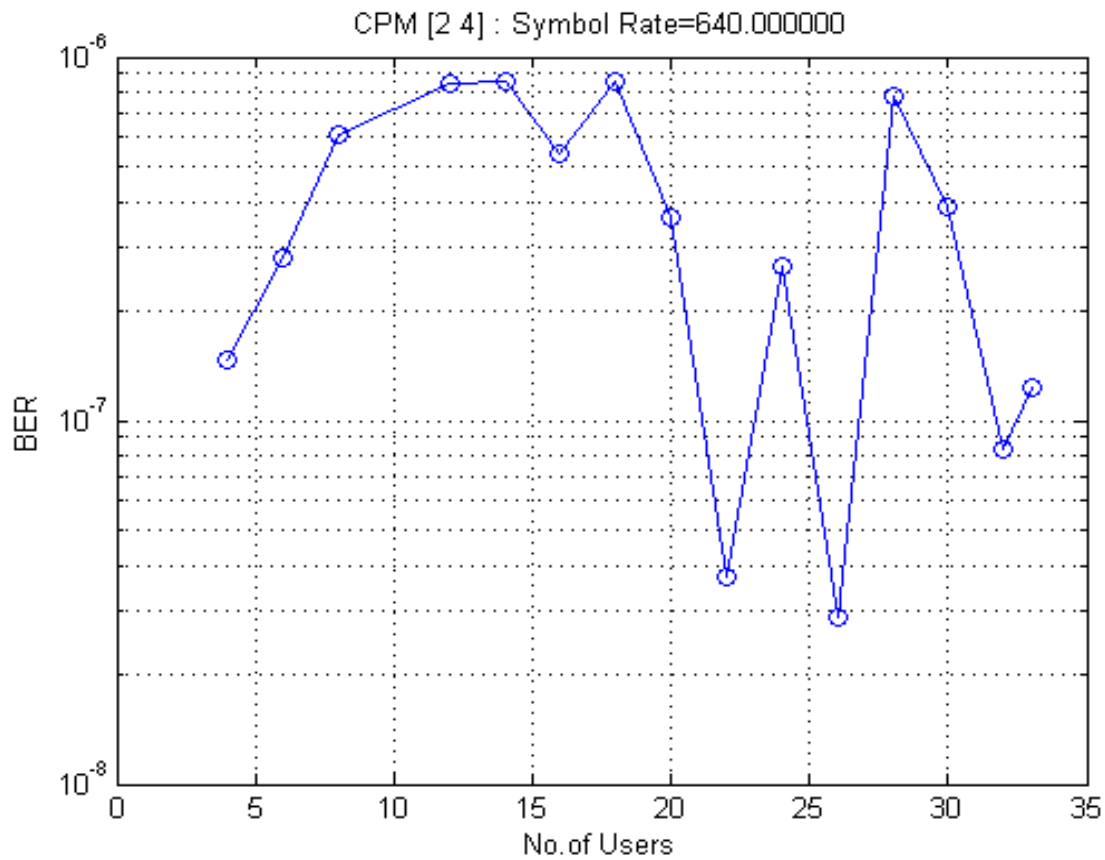

Fig. 14. MC-CDMA System M-ary CPM 


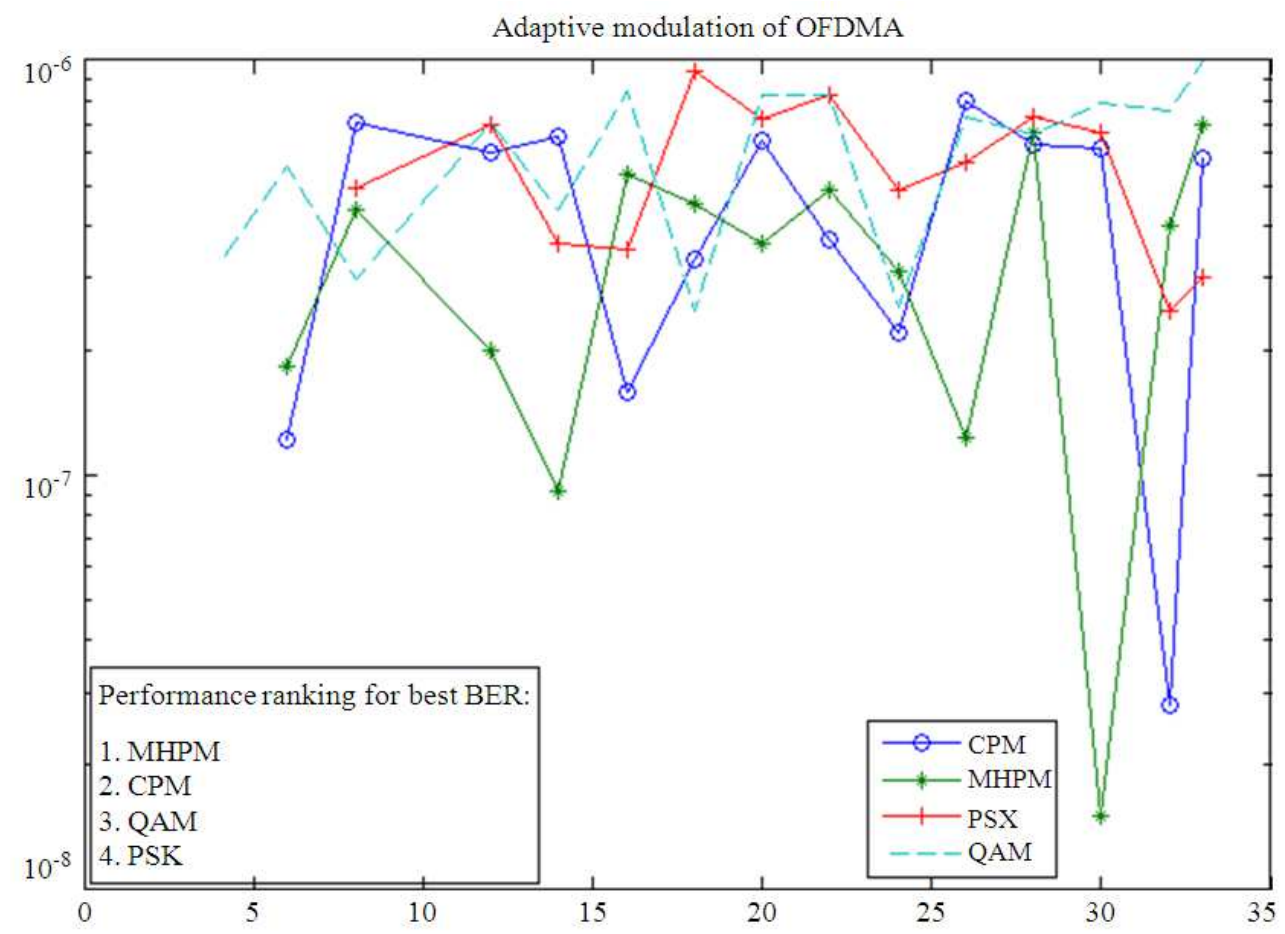

Fig. 15. Comparison of PSK, QAM, CPM and MHPM modulation Schemes for OFDMA system

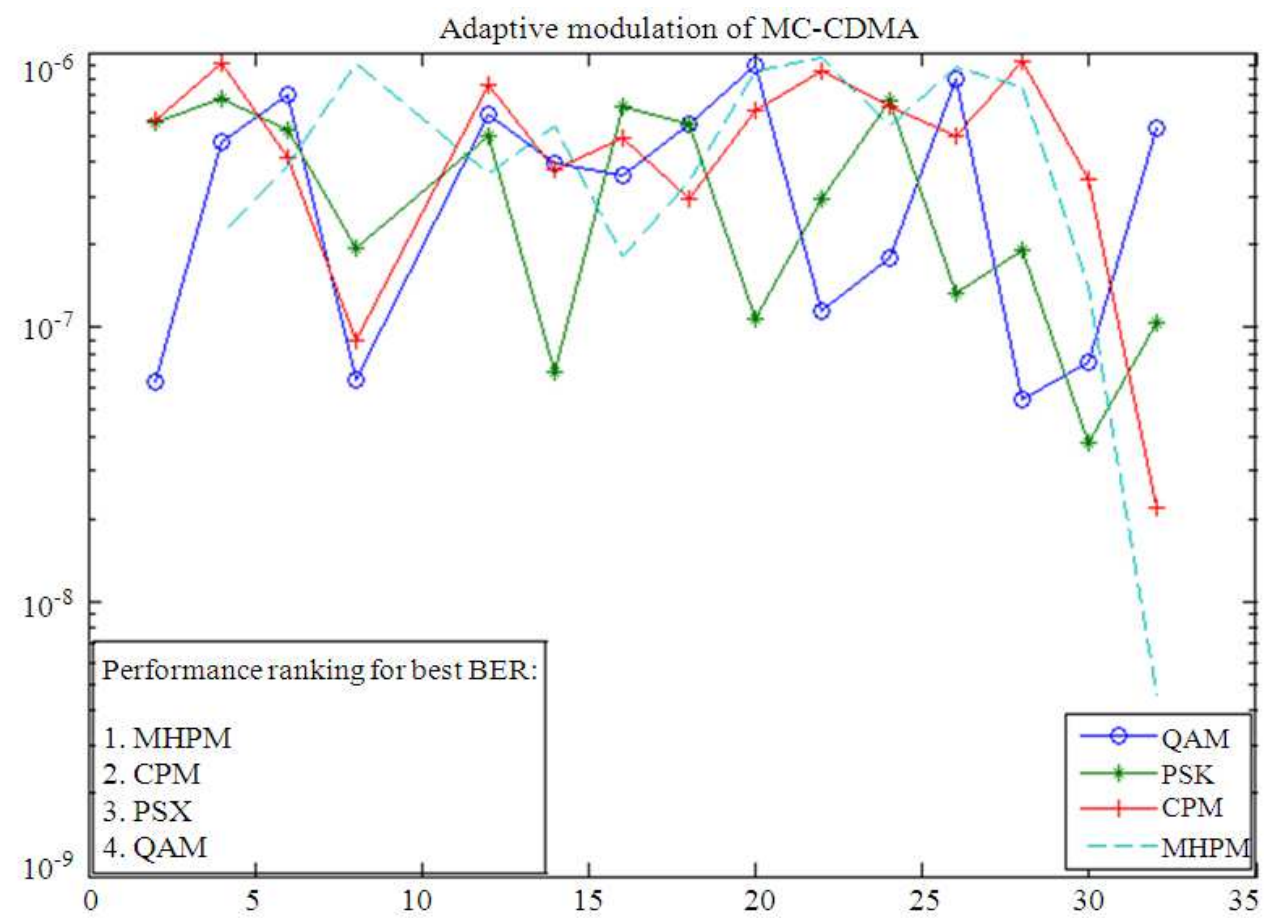

Fig. 16. Comparison of PSK, QAM, CPM and MHPM modulation schemes for MC-CDMA system 


\subsection{MC-CDMA System Mary-PSK, M-ary QAM}

Mary-CPM and M-ary-MHPM Systems are shown in the following Fig. 11-14.

\subsection{OFDMA System and MC-CDMA System}

The BER Vs No of User's of various Adaptive modulation techniques for both OFDMA system and MC-CDMA system are plotted for up to 32 users. The BDBP is constantly maintained at $640 \mathrm{ksymbols} / \mathrm{sec}$. The Comparative graphs are shown in the Fig. 15 and 16.

\section{MATERIALS AND METHODS}

The OFDMA and MC-CDMA transmitter and receiver are designed by using the above parameters given in Table 1-3. The 1/3 rated Turbo coder is selected in transmitter. The symbol rate of $640 \mathrm{ksymbols} / \mathrm{sec}$ is maintained and the simulation is performed using Mat lab and Simulink software (Chatterjee et al., 2003).

\section{RESULTS AND DISCUSSION}

From the graphical analysis, it is clear that the MaryCPM and M-aryMHPM modulation techniques are providing BER of up to $10^{-8}$ as compare to M-ary PSK and M-ary QAM which can provide a BER result of up to $10^{-5}$. From the graph it is found that For OFDMA system, the modulation techniques ranks in following hierarchy of MHPM, CPM, QAM and PSK where as for MC-CDMA system, the modulation techniques ranks as MHPM, CPM, PSK and QAM.

The simulation is performed using Mat lab and Simulink software and the simulation results for OFDMA and MC-CDMA system are plotted for Mary PSK, M-ary QAM. Mary CPM and M-ary MHPM for up to 32 users for symbol rate of $640 \mathrm{ksymbols} / \mathrm{sec}$. The number of users Vs BER curves are plotted for all the modulation techniques of MC-CDMA system and OFDMA System. The overall comparative graph for both MC-CDMA system and OFDMA system are plotted. From both the comparative graph it is found that M-ary MHPM modulation technique is found to be the best Adaptive modulation technique for both MC-CDMA and OFDMA system.

\section{CONCLUSION}

Adaptive modulation based MC-CDMA System or OFDMA System using Turbo encoder is taken in Rayleigh fading environment and the simulation result is plotted. The BER for Mary PSK, M-ary QAM, Mary MHPM and M-ary CPM for BDBP (Bit Duration Bandwidth Product) $640 \mathrm{ksymbols} / \mathrm{sec}$ is analyzed.

It is found that M-ary MHPM has very low BER of up to $10^{-7}$. It is found that the dynamic switching technique of Adaptive modulation has made the BER to be maintained within the lowest limit. The Turbo coder with a rating of $1 / 3$ has made the system to work in adhoc environment very effectively and maintain a constant BER.

So it is concluded that MHPM based Adaptive modulation system with $1 / 3$ \{ne by three\} rated Turbo coder seems to be the best suited modulation system for both MC-CDMA and OFDMA accessing techniques.

\section{REFERENCES}

Chatterjee, S., W.A.C. Fernando and M.K. Wasantha, 2003. Adaptive modulation based MC-CDMA systems for $4 \mathrm{G}$ wireless consumer applications. IEEE Trans. Consumer Electr., 49: 995-1003. DOI: 10.1109/TCE.2003.1261187

Chen, J. and Y. Wang, 2001. Adaptive MLSE equalizers with parametric tracking for multipath fast-fading channels. IEEE Trans. Commun., 49: 655-663. DOI: 10.1109/26.917772

Keller, T. and L. Hanzo, 1998. Adaptive orthogonal frequency division multiplexing schemes. Proceedings of the Adaptive Orthogonal Frequency Division Multiplexing Schemes, (ACTS' 98), Rhodos, Greece, pp: 794-799.

Keller, T. and L. Henzo, 2000. Adaptive modulation techniques for duplex OFDM transmission. IEEE Tran. Vehicular Technol., 49: 1893-1906. DOI: 10.1109/25.892592

McCormick, A.C. and E.A. Al Susa, 2002. Multicarrier CDMA for Future generation mobile communication. Electr. Commun. Eng. J., 14: 52-60. DOI: 10.1049 /ecej: 20020202

Rajwani, I., P.U. Dere and A. Deshpande, 2012. OFDMA based communication system with low peak average power ratio. Int. J. Eng. Res. Applic., 2: $1876-1879$. 
Steele, R. and W. Webb, 1991. Variable rate QAM for data transmissions over Rayleigh fading channels. Proceedings of the Wireless, (Wireless' 91), Calgary, Canada, pp: 1-14.

Wasantha, M.K. and W.A.C. Fernando, 2002. Adaptive modulation techniques for COFDM-CDMA based wireless networks. Proceedings of Wireless Personal Multimedia Communication, (WPMC' 02), Honolulu, Hawaii.
Wasantha, M.K., 2000. Adaptive modulation techniques for OFDM-CDMA based 4G mobile networks. Master Thesis, Asian Institute of Technology, Thailand.

Watson, D., 2000. Mood and Temperament. 1st Edn., Guilford Press, New York, ISBN-10: 1572305266, pp: 340.

Xiong, F., 2006. Digital Modulation Techniques. 2nd Edn., Artech House, Boston, MA., ISBN-10: 1580538630, pp: 1017. 